\title{
The Hawking energy on photon surfaces
}

\author{
Ingemar Bengtsson ${ }^{1}$
}

Received: 31 January 2020 / Accepted: 16 May 2020 / Published online: 25 May 2020

(c) The Author(s) 2020

\begin{abstract}
The Hawking energy has a monotonicity property under the inverse mean curvature flow on totally umbilic hypersurfaces with constant scalar curvature in Einstein spaces. It grows if the hypersurface is spacelike, and decreases if it is timelike. Timelike examples include Minkowski and de Sitter hyperboloids, and photon surfaces in Schwarzschild.
\end{abstract}

Keywords Quasi-local energy · Hawking energy · Photon surfaces

\section{Contents}

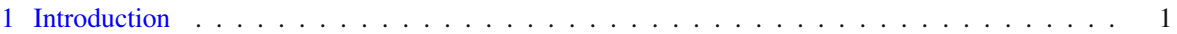

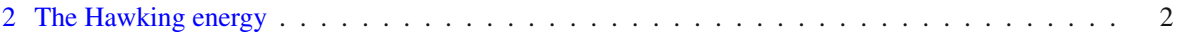

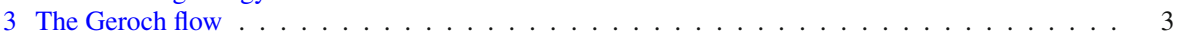

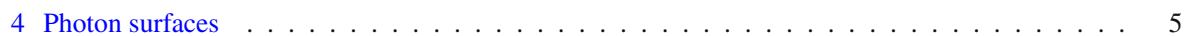

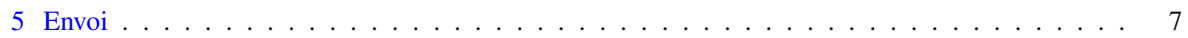

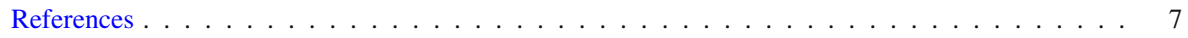

\section{Introduction}

Without much fanfare, Hawking proposed a measure of the energy associated to a closed surface in spacetime [1]. We will explain it below, and refer elsewhere for more details [2-4]. It is agreed that Hawking's expression does not have all the properties one expects 'energy' to have, but it does have the single most important property of that concept: it is useful. Notably, an argument initiated by Geroch [5] and finalized by Huisken and Ilmanen [6] uses the Hawking energy of spheres embedded in a timesymmetric hypersurface to prove the Riemannian Penrose inequality. This is a great improvement in our understanding of energy in general relativity. The idea is to set up a geometric flow that moves any sphere to a large round sphere close to infinity, while

Ingemar Bengtsson

ingemar@fysik.su.se

1 AlbaNova, Fysikum, Stockholms Universitet, 10691 Stockholm, Sweden 
all the time increasing the Hawking energy. From this point of view the occurence of negative Hawking energies in Minkowski space is not a drawback, it is a virtue. One can dream of a generalization of the Geroch flow to spacetime leading to a proof of the Penrose inequality in full generality [7], but then one has to deal with the fact that Minkowski space also contains spheres with positive Hawking energy [8]. Hence there must be a subtle story to tell about the flow and any monotonicity property that the Hawking energy has, if the dream is to come true.

Our ambitions in this note are quite modest. First we show that the Hawking energy is never positive on spacelike hyperboloids in Minkowski space, and never negative on timelike hyperboloids. (With a suitable definition the same holds for de Sitter space.) Then we show heuristically that, in Einstein spaces, the Hawking energy is monotone under Geroch's inverse mean curvature flow on any totally umbilic hypersurface with constant curvature. In particular it cannot increase if that hypersurface is timelike. Timelike totally umbilic hypersurfaces are known as photon surfaces, because they are swept out by light rays emitted tangentially from an embedded surface [9]. Spacetimes admitting complete photon surfaces are quite rare [10], but spherically symmetric spacetimes offer obvious examples (because then one can choose a round sphere to emit light rays from). There has been quite a bit of interest in photon surfaces recently [11-13]. The papers by Cederbaum et al. are particularly relevant for us [14-16]. The photon surfaces that fill the Schwarzschild exterior have constant scalar curvature [14], so that they provide examples to which our observation about the monotonicity of the Hawking energy applies.

\section{The Hawking energy}

Since we will deal with topological 2-spheres embedded in hypersurfaces that are themselves embedded in spacetime, there will be a bit of a strain on the notation. We will use $R_{S}, \bar{R}$, and $R$ for the scalar curvature of respectively the spheres, the hypersurfaces, and the spacetime, and we use $\gamma_{a b}, \bar{g}_{a b}$, and $g_{a b}$ for their respective first fundamental forms. The normal vector of a hypersurface is denoted by $\vec{e}$, and the normal vector of a surface within a hypersurface by $\vec{n}$. Timelike normal vectors are assumed to be future directed. The null normals of the surface are therefore

$$
\vec{k}_{ \pm}= \begin{cases}\vec{e} \pm \vec{n} & \text { if the hypersurface is spacelike } \\ \vec{n} \pm \vec{e} & \text { if the hypersurface is timelike. }\end{cases}
$$

The shape tensor of the surface is denoted by the kernel letter $K$, the second fundamental form of a hypersurface is denoted by the kernel letter $\Pi$, and that of the surface within the hypersurface by $p$. The trace of the shape tensor contracted by a normal vector is denoted with a subscript. Thus we will come across formulas such as $K_{n}=p$, meaning that the trace of the shape tensor contracted into the normal vector $\vec{n}$ is equal to the trace of the second fundamental form of the surface within the hypersurface. We do not think that the notation will cause any difficulties, but the fact that the hypersurface can be timelike or spacelike can be confusing. The shape tensor can be split into 


$$
K_{a b}\left(k_{ \pm}\right)=\sigma_{ \pm a b}+\frac{1}{2} \gamma_{a b} \theta_{ \pm}
$$

where we introduced the null expansions $\theta_{ \pm}$and the traceless shears $\sigma_{ \pm a b}$.

The definition of the Hawking energy can now be stated in three equivalent forms:

$$
\begin{aligned}
E_{H}= & \sqrt{\frac{A}{16 \pi}}\left(1+\frac{1}{16 \pi} \oint \theta_{+} \theta_{-} \mathrm{d} S-\frac{\lambda}{3} \frac{A}{4 \pi}\right), \\
E_{H}= & \frac{1}{16 \pi} \sqrt{\frac{A}{16 \pi}}\left(\oint\left(2 R_{S}+\theta_{+} \theta_{-}\right) \mathrm{d} S-\frac{\lambda}{3} \frac{A}{4 \pi}\right), \\
E_{H}= & \frac{1}{8 \pi} \sqrt{\frac{A}{16 \pi}} \\
& \oint\left(\sigma_{+a b} \sigma_{-}^{a b}+\left(G_{a b}+\lambda g_{a b}\right) k_{+}^{a} k_{-}^{b}-\frac{1}{2} C_{a b c d} k_{+}^{a} k_{-}^{b} k_{+}^{c} k_{-}^{d}\right) \mathrm{d} S .
\end{aligned}
$$

Here $A$ is the area of the 2-sphere, $C_{a b c d}$ is the Weyl tensor, and $G_{a b}$ the Einstein tensor. The term proportional to the cosmological constant $\lambda$ was added to Hawking's definition as an afterthought [17]. To go between (3) and (4), apply the Gauss-Bonnet theorem. To go between (4) and (5), use the Gauss formulas in the codimension 2 case.

From (5) we see immediately that the Hawking energy vanishes for an arbitrary cut of a lightcone in Minkowski and de Sitter space, because in these cases all the curvature terms and one of the shear tensors vanish. We can also make use of

$$
\sigma_{+a b} \sigma_{-}^{a b}= \begin{cases}\sigma_{e a b} \sigma_{e}^{a b}-\sigma_{n a b} \sigma_{n}^{a b} & \text { if the hypersurface is spacelike } \\ \sigma_{n a b} \sigma_{n}^{a b}-\sigma_{e a b} \sigma_{e}^{a b} & \text { if the hypersurface is timelike. }\end{cases}
$$

A totally umbilic hypersurface is defined as one whose second fundamental form is everywhere shear-free. (The strange name 'umbilic' is due to the fact that the surface of the human body has a shear-free second fundamental form at the centre of the navel.) On such a hypersurface it holds that

$$
\sigma_{e a b}=0
$$

Inspection of formula (5) then shows that the Hawking energy is never positive for arbitrary spheres in a totally umbilic spacelike hypersurface in a conformally flat Einstein space, and never negative if the hypersurface is timelike. On a timelike static cylinder both signs can occur $[3,8]$. For a round sphere in the Schwarzschild spacetime the Hawking energy evaluates to $m$, the total mass of the spacetime.

\section{The Geroch flow}

Geroch's idea was to move a sphere within a hypersurface, in the direction of its normal vector $\vec{n}$ and with a speed that depends on the extrinsic curvature of the sphere 
[5]. When doing so the metric and the mean curvature of the surface change according to

$$
\begin{aligned}
\dot{\gamma}_{i j} & =\mathcal{L}_{\phi \vec{n}} \gamma_{i j}=2 \phi p_{i j} \\
\dot{p} & =-\Delta_{S} \phi-\frac{1}{2} \phi\left(p_{i j} p^{i j}+p^{2}\right)+\frac{\epsilon}{2} \phi\left(R_{S}-\bar{R}\right) .
\end{aligned}
$$

The formula for $\dot{p}$ is the formula for the second variation of the area rewritten using the Gauss' equation, $\Delta_{S}$ is the intrinsic Laplacian, and $\epsilon=\vec{n}^{2}$. This gives us another sign to remember,

$$
\epsilon= \begin{cases}+1 & \text { if the hypersurface is spacelike } \\ -1 & \text { if the hypersurface is timelike. }\end{cases}
$$

For the rate of the flow Geroch sets

$$
\phi=\frac{1}{p}
$$

which is why his flow is referred to as the inverse mean curvature flow. With this choice $\dot{A}=A$. (To learn about curvature flows in general, consult Sethian [18].) Geroch showed heuristically that the Hawking energy has an important monotonicity property if the flow takes place within a time symmetric hypersurface. If $p=0$ at some point of the surface the flow can only exist in a suitable weak sense, which is why it took so long to turn Geroch's arguments into a rigourous theorem [6].

There have been many studies of the monotonicity properties of the Hawking energy in more general situations $[2,8,19]$. Here we will apply the Geroch flow to a sphere within a totally umbilic hypersurface inside an Einstein space, but we allow the hypersurface to be timelike. Consider the Hawking energy in version (4), that is

$$
E_{H}=\frac{1}{16 \pi} \sqrt{\frac{A}{16 \pi}} \oint\left(2 R_{S}+\epsilon K_{e}^{2}-\epsilon K_{n}^{2}-\frac{4 \lambda}{3}\right) \mathrm{d} S
$$

We make use of

$$
K_{e}=\gamma^{a b} \Pi_{a b}=\frac{1}{3} \Pi \gamma^{a b} \bar{g}_{a b}=\frac{1}{3} \Pi\left(\bar{g}^{a b}-n^{a} n^{b}\right) \bar{g}_{a b}=\frac{2}{3} \Pi .
$$

Recall that $\bar{g}_{a b}$ and $\gamma_{a b}$ are the first fundamental forms of the hypersurface and the surface, respectively. We also use the equation $K_{n}=p$. The Gauss equation combined with the Einstein equation implies

$$
\epsilon \bar{R}+\frac{2}{3} \Pi^{2}=2 G_{a b} e^{a} e^{b}=2 \epsilon \lambda
$$


Then the Hawking energy is

$$
E_{H}=\frac{1}{16 \pi} \sqrt{\frac{A}{16 \pi}} \oint\left(2 R_{S}-\epsilon p^{2}-\frac{2}{3} \bar{R}\right) \mathrm{d} S .
$$

We now repeat Geroch's calculation [5]. We only have an extra sign and an extra term involving $\bar{R}$ to keep track of. The result is

$$
\dot{E}_{H}=\frac{1}{16 \pi} \sqrt{\frac{A}{16 \pi}}\left(\epsilon C-\frac{2}{3} \oint \dot{\bar{R}} \mathrm{~d} S\right)
$$

where

$$
C=\oint\left(\frac{2}{p^{2}} D_{i} p D^{i} p+\left(p_{i j}-\frac{p}{2} \gamma_{i j}\right)\left(p^{i j}-\frac{p}{2} \gamma^{i j}\right)\right) \mathrm{d} S \geq 0
$$

If the scalar curvature $\bar{R}$ of the hypersurface is constant so that $\dot{\bar{R}}=0$ then the Hawking energy is a monotone quantity. It can only increase if $\epsilon=+1$, and it can only decrease if $\epsilon=-1$.

The derivation is heuristic because it leaves open the question whether the flow exists. On spacelike hypersurfaces this is a hard question [6]. Surfaces embedded in timelike hypersurfaces are in many ways less wild than those one finds embedded in spacelike hypersurfaces, but on a timelike hyperboloid in Minkowski space we do have the problem that through every point there passes a surface with $p=0$. (The timelike hyperboloid is maximally symmetric, so it is enough to show this for one point.) But let us consider a round sphere with $p$ positive and constant over the surface, and let us normalize the hyperboloid in which it sits so that $\bar{R}=6$. A quick calculation confirms that $p<2$ for such a surface, and another quick calculation that

$$
\dot{p}=\frac{2}{p}-\frac{p}{2}=\frac{(2-p)(2+p)}{2 p}>0 .
$$

This evolves towards $p=2$. Although this is a special case it seems clear that surfaces whose mean curvatures are everywhere positive will flow to round spheres at $\mathscr{I}$ without encountering any special problems. The problem with $p=0$ will loom very large in the Schwarzschild photon sphere at $r=3 m$, but that is a very special case since the photon sphere never reaches $\mathscr{I}$.

\section{Photon surfaces}

All the photon surfaces in the Schwarzschild spacetime, not just the photon sphere, have constant scalar curvature [14]. This provides a reasonably large set of examples for which the Hawking mass decreases under the inverse mean curvature flow, and it is worthwhile to see exactly how it happens. 
We start with the metric

$$
d s^{2}=-V(r) d t^{2}+\frac{d r^{2}}{V(r)}+r^{2} d \theta^{2}+r^{2} \sin ^{2} \theta d \phi^{2}, \quad V(r)>0 .
$$

We define a spherically symmetric timelike hypersurface through

$$
r=r(t) \text {. }
$$

We denote its first fundamental form by $g_{a b}$, and define

$$
F(r, \dot{r})=V(r)-\frac{\dot{r}^{2}}{V(r)}>0
$$

(Now the dot notation refers to differentiation with respect to $t$.) The second fundamental form is quickly computed to be

$$
\Pi_{a b}=\frac{1}{\sqrt{F}} \frac{V}{r} g_{a b}+\frac{1}{\sqrt{F}}\left(\frac{V^{2}}{r}-\frac{V V_{, r}}{2}-\left(\frac{1}{r}-\frac{3}{2} \frac{V_{, r}}{V}\right) \dot{r}^{2}-\ddot{r}\right) \nabla_{a} t \nabla_{b} t .
$$

The hypersurface is a photon surface if and only if the expression within brackets vanishes. This gives a second order differential equation for $r(t)$ which can be derived from a Lagranian. In principle it can be solved using quadratures, because it admits the conserved quantity

$$
\frac{r^{2}}{V}-\frac{r^{2} \dot{r}^{2}}{V^{3}}=-2 E=r_{0}^{2}
$$

The different cases that arise are discussed by Cederbaum and et al. [15,16]. We can use the information we already have to compute the mean curvature

$$
\Pi=g^{a b} \Pi_{a b}=\frac{3}{r_{0}}
$$

and (somewhat more labouriously) the scalar curvature

$$
\bar{R}=\frac{6}{r_{0}^{2}}+\frac{2}{r^{2}}\left(1-V-V_{, r}\right)
$$

of the photon surface.

So far we have not imposed Einstein's equations. When we do we find that

$$
V(r)=1-\frac{2 m}{r}-\frac{\lambda}{3} r^{2}
$$


and we have arrived at the Schwarzschild-de Sitter spacetime. In this case we find that

$$
\bar{R}=\frac{6}{r_{0}^{2}}+2 \lambda,
$$

a constant value for all our photon surfaces. Indeed, in the Einstein case this follows directly from the fact that the mean curvature is constant.

\section{Envoi}

It is comforting that we have a set of examples to which our observation about the Hawking energy applies. Of course it has to be admitted that the Schwarzschild spacetime is a very special spacetime. The Penrose inequality holds in spherical symmetry [20,21], and we are not able to offer any suggestions about how to move beyond that. But we have seen that the Hawking energy has many subtle properties. Building intuition for them is likely to be useful.

Acknowledgements Open access funding provided by Stockholm University. I thank Patrik Lindberg and José Senovilla for sharing their many insights, and Carla Cederbaum for sketching the content of reference [16]. And I happily acknowledge the Mittag-Leffler Institute in Djursholm, Stockholm, for a wonderful relativity program.

Open Access This article is licensed under a Creative Commons Attribution 4.0 International License, which permits use, sharing, adaptation, distribution and reproduction in any medium or format, as long as you give appropriate credit to the original author(s) and the source, provide a link to the Creative Commons licence, and indicate if changes were made. The images or other third party material in this article are included in the article's Creative Commons licence, unless indicated otherwise in a credit line to the material. If material is not included in the article's Creative Commons licence and your intended use is not permitted by statutory regulation or exceeds the permitted use, you will need to obtain permission directly from the copyright holder. To view a copy of this licence, visit http://creativecommons.org/licenses/by/4.0/.

\section{References}

1. Hawking, S.W.: Gravitational radiation in an expanding universe. J. Math. Phys. 9, 598 (1968)

2. Eardley, D.M.: Global problems in numerical relativity. In: Smarr, L.L. (ed.) Sources of Gravitational Radiation. Cambridge UP, Cambridge (1979)

3. Szabados, L.B.: Quasi-local energy-momentum and angular momentum in GR: a review article. Liv. Rev. Relativ. 7, 4 (2004)

4. Bray, H.L., Roesch, H.P.: Null geometry and the Penrose conjecture. arXiv:1708.00941

5. Geroch, R.: Energy extraction. Ann. N. Y. Acad. Sci. 224, 108 (1973)

6. Huisken, G., Ilmanen, T.: The inverse mean curvature flow and the Riemannian Penrose inequality. J. Differ. Geom. 59, 353 (2001)

7. Frauendiener, J.: On the Penrose inequality. Phys. Rev. Lett. 87, 101101 (2001)

8. Bray, H.L., Jauregui, J.L., Mars, M.: Time flat surfaces and the monotonicity of the spacetime Hawking mass II. Ann. Henri Poincaré 17, 1457 (2016)

9. Claudel, C.-M., Virbhadra, K.S., Ellis, G.F.R.: The geometry of photon surfaces. J. Math. Phys. 42, 818 (2001)

10. Perlick, V.: On totally umbilic submanifolds of semi-Riemannian manifolds. Nonlinear Anal. 63, 511 (2005) 
11. Foertsch, T., Hasse, W., Perlick, V.: Inertial forces and photon surfaces in arbitrary spacetimes. Class. Quantum Gravity 20, 4635 (2003)

12. Khoo, F.S., Ong, Y.C.: Lux in obscuro: photon orbits of extremal black holes revisited. Class. Quantum Gravity 33, 235002 (2016)

13. Gibbons, G.W., Warnick, C.M.: Aspherical photon and anti-photon surfaces. Phys. Lett. B 763, 169 (2016)

14. Cederbaum, C.: Uniqueness of photon spheres in static vacuum asymptotically flat spacetimes. In: Agranovsky, M.L., et al. (eds) Complex Analysis and Dynamical Systems VI, Contemporary Mathematics, vol. 667, p. 86. AMS, Providence (2015)

15. Cederbaum, C., Galloway, G.J.: Photon surfaces with equipotential time slices. arXiv:1910.04220

16. Cederbaum, C., Jahns, S., Vičánek Martínez, O.: In preparation (2020)

17. Boucher, W., Gibbons, G.W., Horowitz, G.T.: Uniqueness theorems for anti-de Sitter spacetime. Phys. Rev. D 30, 2447 (1984)

18. Sethian, J.A.: Level Set Methods and Fast Marching Methods. Cambridge UP, Cambridge (1999)

19. Bray, H., Hayward, S., Mars, M., Simon, W.: Generalized inverse mean curvature flows in spacetime. Commun. Math. Phys. 272, 119 (2007)

20. Malec, E., Murchadha, N.Ó.: Trapped surfaces and the Penrose inequality in spherically symmetric geometries. Phys. Rev. D 49, 6931 (1994)

21. Husain, V., Singh, S.: On the Penrose inequality in anti-de Sitter space. Phys. Rev. D 96, 104055 (2017)

Publisher's Note Springer Nature remains neutral with regard to jurisdictional claims in published maps and institutional affiliations. 